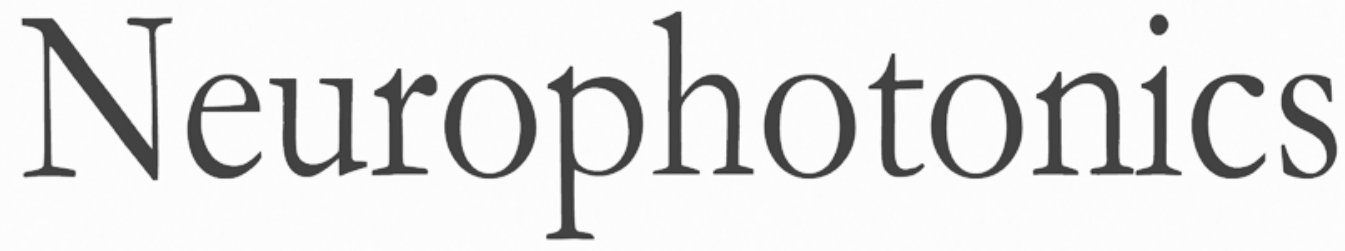

Differential efficiency of transcutaneous electrical nerve stimulation in dominant versus nondominant hands in fibromyalgia: placebo-controlled functional nearinfrared spectroscopy study

\author{
Aykut Eken \\ Murat Kara \\ Bora Baskak \\ Ayşegül Baltacı \\ Didem Gökçay
}




\title{
Differential efficiency of transcutaneous electrical nerve stimulation in dominant versus nondominant hands in fibromyalgia: placebo-controlled functional near-infrared spectroscopy study
}

\author{
Aykut Eken, ${ }^{a, \star}$ Murat Kara, ${ }^{b}$ Bora Baskak, ${ }^{c, d}$ Ayşegül Baltacı, ${ }^{e}$ and Didem Gökçay ${ }^{f}$ \\ aüzce University, Faculty of Engineering, Biomedical Engineering Department, Düzce, Turkey \\ ${ }^{b}$ Hacettepe University Medical School, Department of Physical and Rehabilitation Medicine, Ankara, Turkey \\ ${ }^{\mathrm{c} A n k a r a}$ University Medical School, Department of Psychiatry, Ankara, Turkey \\ ${ }^{\mathrm{d} A n k a r a}$ University, Brain Research and Application Center, Ankara, Turkey \\ 'Yenimahalle Education and Research Hospital, Department of Physical and Rehabilitation Medicine, Ankara, Turkey \\ ${ }^{\mathrm{f}}$ Middle East Technical University, Informatics Institute, Department of Health Informatics, Ankara, Turkey
}

\begin{abstract}
Using functional near-infrared spectroscopy (fNIRS), modulation of hemodynamic responses by transcutaneous electrical nerve stimulation (TENS) during delivery of nociceptive stimulation was investigated in fibromyalgia (FM) patients and healthy controls for both hands. Two experiments were conducted: (1) median nerve stimulation with TENS and (2) painful stimulation using electronic von Frey filaments with TENS/placebo TENS. Mean $\Delta \mathrm{HbO}_{2}$ brain activity was compared across groups and conditions using factorial ANOVA. Dominant (right) hand stimulation indicated significant interactions between group and condition in both hemispheres. Post hoc results revealed that FM patients showed an increased activation in "pain + TENS" condition compared to the "pain + placebo TENS" condition while the brain activity patterns for these conditions in controls were reversed. Left-hand stimulation resulted in similar TENS effects (reduced activation for "pain + TENS" than "pain + placebo TENS") in both groups. TENS effects in FM patients might be manipulated by the stimulation side. While the nondominant hand was responsive to TENS treatment, the dominant hand was not. These results indicate that stimulation side might be an effective factor in FM treatment by using TENS. Future studies are needed to clarify the underlying mechanism for these findings. @ 2017 Society of Photo-Optical Instrumentation Engineers (SPIE) [DOI: 10.1117/1.NPh.5.1.011005]
\end{abstract}

Keywords: fibromyalgia; functional near-infrared spectroscopy; von Frey filaments; transcutaneous electrical nerve stimulation; handedness.

Paper 17017SSRR received Jan. 27, 2017; accepted for publication Aug. 10, 2017; published online Sep. 5, 2017.

\section{Introduction}

Fibromyalgia (FM) is a chronic pain syndrome characterized by widespread hyperalgesia. ${ }^{1}$ Its prevalence is about $2 \%$ to $8 \% .^{2}$ It is generally accepted that FM is associated with central sensitization/excitability and decreased pain inhibition due to dysfunction in peripheral and central mechanisms. ${ }^{3}$ Transcutaneous electrical nerve stimulation (TENS), which is used to relieve acute and chronic pain, ${ }^{1,4-7}$ is a nonpharmacological treatment option for FM. Although its actual mechanism is not well known, according to the "gate control theory of pain," 8 it is postulated that TENS prevents the conduction of nociceptive afferent fibers $(A \delta, \mathrm{C}$-fibers) activity on the dorsal horn of the spinal cord by activating nonnociceptive $A \beta$ fibers. In addition, recent studies have shown that TENS causes activation in central descending inhibitory pathways from midbrain/brainstem,,${ }^{9,10}$ resulting in a decrease in excitability of nociceptive neurons in the spinal cord. ${ }^{11}$

Cerebral pain processing has been studied in healthy participants by using different modalities measuring hemodynamic activity, such as functional magnetic resonance imaging $(\mathrm{fMRI})^{12,13}$ and functional near-infrared spectroscopy (fNIRS). fNIRS is a portable and noninvasive neuroimaging modality

*Address all correspondence to: Aykut Eken, E-mail: ekenaykut@gmail.com that was used in several painful stimulation studies that focused on tonic pain activation in healthy participants, ${ }^{14,15}$ sensorimotor region in chronic back pain patients, ${ }^{16}$ frontal lobe activation during electrical stimulation, ${ }^{17}$ and somatosensory regions during painful heat ${ }^{18,19}$ stimulation. In FM, hemodynamic responses caused by cognitive performance and painful stimulation were compared by using fNIRS. ${ }^{20}$ Pain related activation is generally observed in the primary [postcentral gyrus (post-CG)] (SI) and secondary (SII) somatosensory cortices, anterior cingulate cortex (ACC), and insular cortices that play an important role in pain perception. ${ }^{12,21}$ In addition, an fMRI study in healthy subjects ${ }^{22}$ has shown that cortical activity was decreased during nociceptive stimulus when TENS was applied. However, to our best notice, there is no study evaluating the effects of TENS during the application of nociceptive stimulus in FM.

Moreover, hand dominancy is an important factor for pain processing. There are several neuroimaging studies performed by using noxious laser, ${ }^{23,24}$ electrical stimulation, ${ }^{25}$ and heat stimulation $^{26}$ that deliver nociceptive stimulation to both hands $^{23-26}$ of healthy controls (HC). These studies showed that there is bilateral activation and contralateral bias in SI, SII, insula, and thalamus ${ }^{24,26}$ and also motor output related structures such as putamen and cerebellum. ${ }^{23}$ 
Unquestionably, neuronal activity in these regions is critical to understand the pathophysiology of FM. Recent fMRI studies $^{27-35}$ suggested that FM patients have augmented corti$\mathrm{cal} /$ subcortical pain processing and increased sensitivity to not only nociceptive stimulus but also nonnociceptive stimulus. ${ }^{36,37}$ Nociceptive stimulation application to the nondominant hand indicated that contralateral SI, SII, IPL and in some studies, insula was activated in right-handed FM patients. ${ }^{27,28,30}$ Nociceptive stimulation application to dominant hand revealed that contralateral SI, SII, and bilateral IPL, insula and basal ganglia were activated in right-handed FM patients. ${ }^{33}$ Also, incision-based studies to the dominant hand revealed that righthanded FM patients showed activity in frontal, motor, and cingulate cortices. ${ }^{32,34}$ Furthermore, a recent fNIRS study carried out by applying nociceptive stimulation to the dominant hand of right-handed participants suggested increased bilateral cortical activation in FM patients. ${ }^{20}$ In a recent meta-analysis study, ${ }^{38}$ results showed differential pain processing for righthanded people, such that, pain was more tolerable when presented to the dominant side. To our best knowledge, so far, nociceptive stimulation in FM patients has not been studied during application of TENS by stimulating both hands. In order to reduce variability in hemispheric lateralization, we chose a right-handed patient population and matched controls while investigating the TENS effect in both hands of FM patients.

We used fNIRS, a portable/inexpensive system utilized in several neuroimaging studies, ${ }^{39,40}$ to observe the hemodynamic activity caused by nociceptive stimuli, as well as TENS. We expected higher hemodynamic activity in FM patients than HC due to hypersensitivity to painful stimulation caused by central sensitization. ${ }^{41}$ This condition is called "hyperalgesia:" excessive response to the painful stimulation. For both hands of patient and HC groups, we hypothesized that when TENS is used along with painful stimulation, the hemodynamic activity would decrease.

\section{Materials and Methods}

Nineteen right-handed FM patients fulfilling the American College of Rheumatology 1990 criteria for $\mathrm{FM}^{42}$ and 17 right-handed HC were enrolled. All subjects stopped the intake of analgesic drugs at least $12 \mathrm{~h}$ before the experiment. Age, gender, beck depression inventory (BDI), menstruation cycle, fibromyalgia impact questionnaire (FIQ), and tender point count were recorded. All subjects were informed about the study protocol, which was approved by the Ethical Board Committee of Ankara University (No. 04-178-14). The study procedures were carried out abiding by the Helsinki declaration and subjects gave signed informed consent to participate.

\subsection{Pain Threshold Measurements}

To determine the amount of nociceptive stimuli to be delivered during the experiment, we assessed individual pain thresholds obtained from each thumb of each subject. We applied a quantitative sensory testing (QST) method by using electronic von Frey (eVF) anesthesiometer (Ugo Basile Co., Italy), a precise and accurate method for pain threshold application. ${ }^{43,44}$ In the QST method with eVF, a continuously variable force transducer using a rigid filament directly contacted the thumb, and an increased mechanical pressure was applied five times with increasing force of $0.1 \mathrm{~g}$ steps by using a single nonflexible filament that has a diameter of $0.5 \mathrm{~mm}$. Stimulus was delivered perpendicularly to the interphalangeal joint of thumbs in every participant. For every five application, while stimulus was applied in a linearly increasing intensity trend, participants gave a verbal sign when they felt an unpleasant feeling caused by sharp painful sensation. ${ }^{44}$ This pressure value was noted as the least pressure intensity in which participants perceived pain. ${ }^{45}$ Between every measurement, there was a 20 -s interval to prevent habituation. Then, these five measurements were averaged and recorded as individual pressure pain threshold.

\subsection{Channel Positioning and the fNIRS System}

In our study, fNIRS was performed at Ankara University Brain Research Center with Hitachi ETG 4000 continuous wave fNIRS system (Hitachi Co., Japan). Optical near-infrared lights with two different wavelengths (695 and $830 \mathrm{~nm}$ ) were sent to the head surface via a source optode and captured back by a detector optode. Sampling frequency was $10 \mathrm{~Hz}$. Optical light signals were converted to $\Delta \mathrm{HbO}_{2}$ and $\Delta \mathrm{Hb}$ by using modified Beer-Lambert law. ${ }^{46}$ We used the $2 \times 3 \times 3$ optode configuration including 24 channels.

We utilized the electroencephalography (EEG) 10-20 electrode positioning system to position the fNIRS source and detectors onto the head surface. ${ }^{47}$ In this system, half of the distance from nasion to inion corresponds to the channel $\mathrm{C} z$. After defining the position of $\mathrm{C} z$, we set the $3 \times 3$ probe holders for each hemisphere over the line passing through both ears. Then, we defined positions of $\mathrm{C} 3$ and $\mathrm{C} 4$ by measuring the distance between both tragi, and $30 \%$ of this value gave us the position of $\mathrm{C} 3$ from left tragus and $\mathrm{C} 4$ from the right tragus, which corresponded to the left and right SI, respectively, as described previously. ${ }^{48,49}$ Source numbers 18 and 13 were placed onto the points $\mathrm{C} 3$ and $\mathrm{C} 4$ in both hemispheres, respectively (Fig. 1). After the probe holder placement, we marked optode positions by using a three-dimensional digitizer (Polhemus Co., Vermont) to determine the exact position of each channel. Then, we obtained the position file to use it for registering to the Montreal Neurological Institute (MNI) space to determine the landmarks that correspond to every channel position by using NIRS analysis package. ${ }^{50}$ Then, we averaged coordinate values

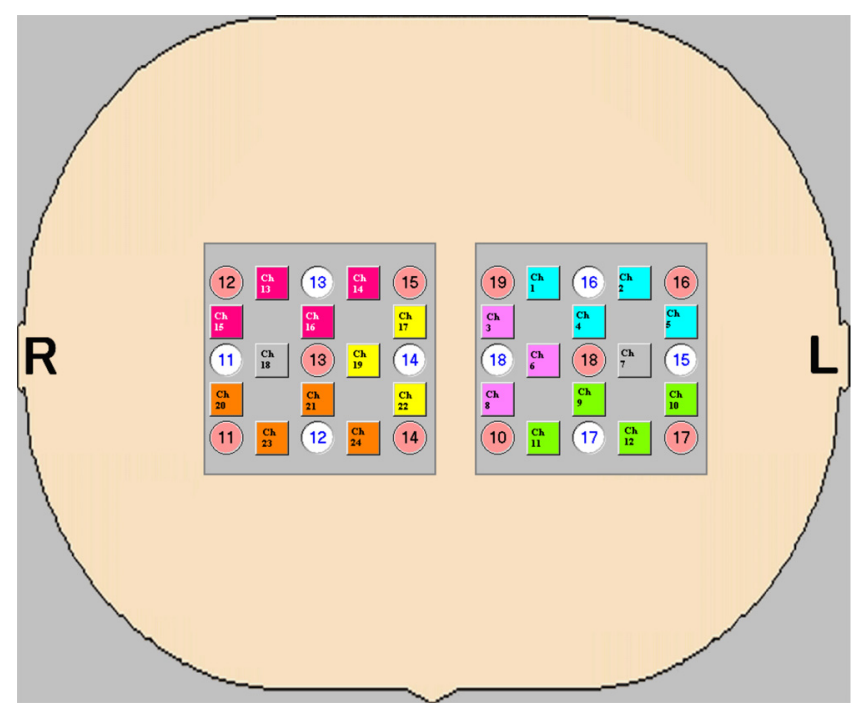

Fig. 1 Channel and optode configuration of $2 \times 3 \times 3$ probe setting. Locations that are represented as squares are channels. White circles that include numbers in blue are detectors. Pink circles that include numbers in black are sources. $\mathrm{R}$, right and $\mathrm{L}$, left. 
Table 1 Channel numbers and average coordinate positions with corresponding cortical structures registered onto MNI space after using LPBA 40 cortical atlas. Probability values were obtained from LPBA 40 cortical atlas. (L, left and $R$, right).

\begin{tabular}{|c|c|c|c|c|c|}
\hline $\begin{array}{l}\text { Channel } \\
\text { number }\end{array}$ & Mean $X$ & Mean $Y$ & Mean $Z$ & SD & $\begin{array}{l}\text { Corresponding } \\
\text { cortical } \\
\text { structure }\end{array}$ \\
\hline 1 & -40.77 & -48.77 & 64.97 & 10.48 & L SPG \\
\hline 2 & -59.55 & -49.44 & 44.52 & 9.41 & $L A G$ \\
\hline 3 & -31.02 & -35.25 & 72.91 & 10.9 & L SPG \\
\hline 4 & -53.88 & -33.41 & 56.97 & 9.99 & L SMG \\
\hline 5 & 67.22 & -34.77 & 30.55 & 8.70 & L SMG \\
\hline 6 & -44.22 & -20 & 65.22 & 11.12 & L post-CG \\
\hline 7 & -62.38 & -19.16 & 43.66 & 9.33 & L post-CG \\
\hline 8 & -29.55 & -5.38 & 70.33 & 11.96 & L pre-CG \\
\hline 9 & -52.63 & -5.02 & 52.94 & 10.42 & L pre-CG \\
\hline 10 & -65.38 & -5.44 & 25.66 & 9.43 & L post-CG \\
\hline 11 & -39.11 & 9.83 & 59.52 & 11.66 & L MFG \\
\hline 12 & -57.27 & 9.75 & 35.80 & 10.00 & L pre-CG \\
\hline 13 & 61.02 & -50.91 & 43.69 & 8.30 & $\mathrm{R} A \mathrm{~A}$ \\
\hline 14 & 42.13 & -49.30 & 64.44 & 9.31 & R SPG \\
\hline 15 & 69 & -36.58 & 30.69 & 7.94 & R SMG \\
\hline 16 & 56.25 & -34.80 & 56.77 & 9.40 & R SMG \\
\hline 17 & 32.63 & -34.97 & 72.94 & 10.31 & R SPG \\
\hline 18 & 64.94 & -20.97 & 44.27 & 9.36 & R SMG \\
\hline 19 & 46.36 & -20.33 & 65.16 & 10.88 & R post-CG \\
\hline 20 & 68.02 & -6.55 & 27.5 & 8.55 & R post-CG \\
\hline 21 & 55.50 & -6 & 53.02 & 10.47 & R post-CG \\
\hline 22 & 31.94 & -5.11 & 69.75 & 11.50 & R pre-CG \\
\hline 23 & 59.86 & 8.86 & 37.05 & 9.54 & R pre-CG \\
\hline 24 & 42.13 & 9.97 & 59.02 & 10.95 & R MFG \\
\hline
\end{tabular}

of all participants. ${ }^{51}$ To obtain brain regions corresponding to MNI coordinates, we used LONI Probabilistic Brain Atlas. ${ }^{52}$ Channel configuration on head is shown in Fig. 1. Channel numbers and corresponding cortical regions with MNI coordinates are shown in Table 1.

\subsection{Experiment 1: Median Nerve Stimulation with TENS}

We performed two different experiments with left and right thumbs in separate runs. Our first experiment was median nerve stimulation by using TENS. For TENS application, we used the Intelect TENS device (Chattanooga Co., Tennessee) with a pulse width of $60 \mu$ s and $115 \mathrm{~Hz}$ frequency via square Dura-Stick Plus Self-Adhesive electrodes with 5-cm size. A predetermined amplitude threshold $(\approx 30 \mathrm{~mA})$ was applied to each participant, which caused a tingling effect on his/her distal forearms/hands. Experimental paradigm included three consequent blocks [Fig. 2(a)]. After a 20-s "rest" condition, 20-s "TENS" was applied and consequent every 20-s "TENS" condition, 20-s "rest" condition was applied.

\subsection{Experiment 2: Painful Stimulation with TENS}

We performed two different experiments with left and right thumbs in separate runs. A single experiment consisted of six pseudorandom blocks with three conditions: "pain + placebo TENS," "pain + TENS," and "rest." The experimental paradigm is shown in Fig. 2(b). "Pain + placebo TENS" and "pain + TENS" conditions were both applied both for 20 s. "Rest" period was applied for $40 \mathrm{~s}$. The experimental run began with a 50-s "initial rest" period. In "pain + placebo TENS" and "pain + TENS" conditions, we applied nociceptive stimuli to the thumb. In the "pain + TENS" condition, the TENS device was turned on during the administration of nociceptive stimuli by applying the conventional TENS parameters mentioned above. During the "pain + placebo TENS" condition, TENS pads were still on the forearms of participants, but the device was not operating. The participants were blind about the timing of the TENS application. The TENS device was connected during the entire experiment and the participants were unaware of the blocks for which the TENS was turned on. TENS application was shown in Fig. 3.

While applying nociceptive stimuli in both conditions, eVF was pressed four times onto the interphalangeal joint of the thumb. Each time eVF is used, pain delivery lasted 4-s, at the individual pressure pain threshold value, which was previously measured before the experiment. Interstimulus interval (ISI) was $1 \mathrm{~s}$. Hence, each repetition of pain plus ISI took $5 \mathrm{~s}$. Each pain block consisted of four repetitions of pain delivery, summing up to a block of $20 \mathrm{~s}$.

Before the experiment, each participant was informed to estimate pain ratings within a scale of 0 (no pain) to 100 (extreme pain). After every block, participants were stipulated to verbally quantify their own subjective pain experience according to this scale during resting periods. During the experimental design, we targeted 70 out of 100 as target pain sensation level for both groups. To reach this level, we applied different nociceptive stimulations for each participant, based on the predetermined individual pain threshold. This type of experiment was previously applied in several studies, referred as "subjective pain control condition., 27,33

\subsection{Data Processing}

We used MATLAB for preprocessing (MathWorks, Inc., Natick, Massachusetts). Preprocessing pipeline included baseline correction, detrending to eliminate low frequency drift, filtering for removal of systemic artifacts, and averaging of blocks in the same condition. Initially, baseline correction was applied to detect the activity trend by observing negative peaks in $\Delta \mathrm{Hb}$ and positive peaks in $\Delta \mathrm{HbO}_{2}$ during active blocks. Wavelet-based detrending filter was used to remove this drift. ${ }^{53}$ Finally, a low-pass filter with a cut-off at $0.05 \mathrm{~Hz}$ 


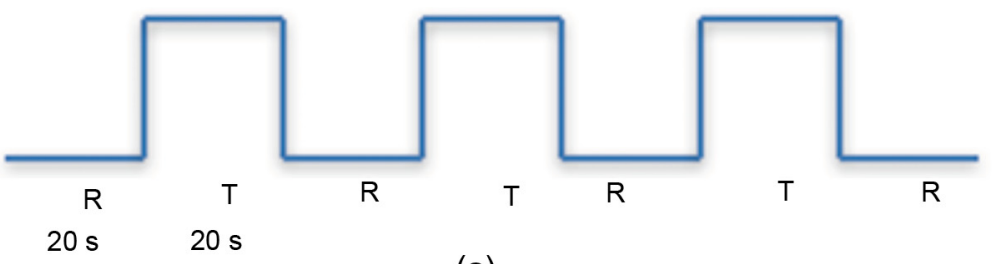

(a)

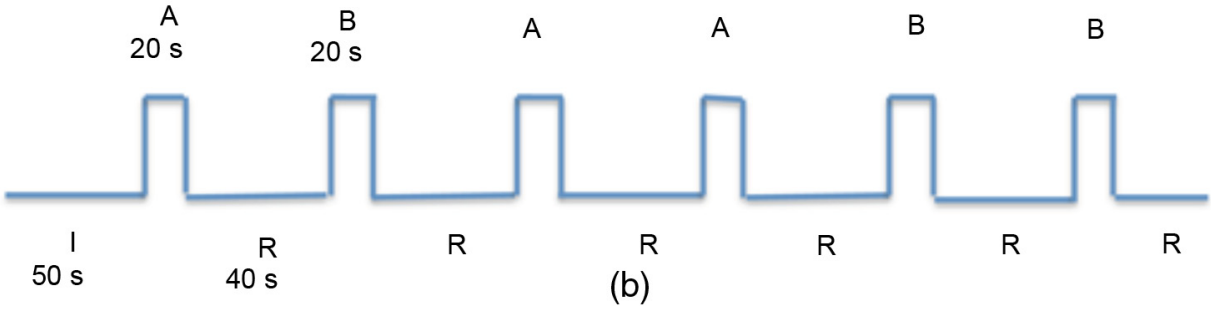

Fig. 2 Experimental design. (a) Median nerve stimulation and (b) painful stimulation with TENS I, initial baseline; A, pain + placebo TENS; B, pain + TENS; R, rest; and T, TENS.

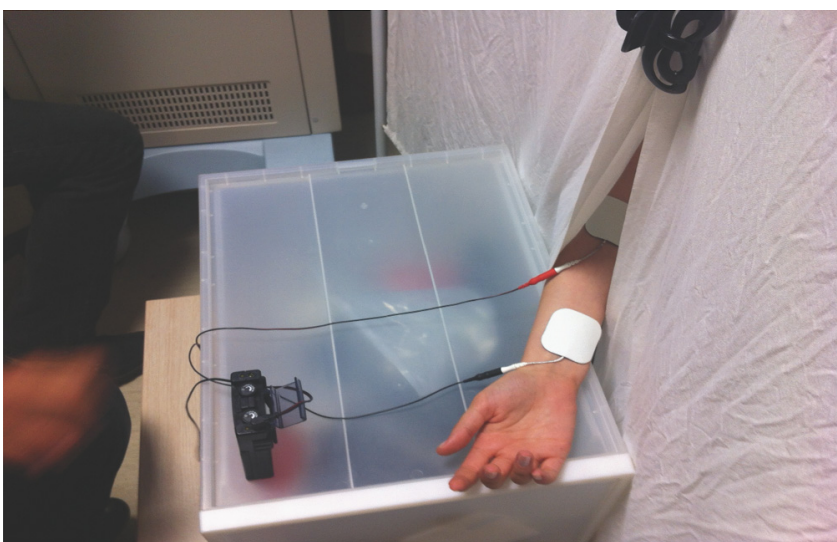

Fig. 3 TENS application to right forearm of a participant.

was applied to remove high-frequency noise that possibly include Mayer waves ( 0.05 to $0.2 \mathrm{~Hz})$, cardiac pulsations $(0.6$ to $2.0 \mathrm{~Hz})$, and respiratory artefact $(0.15$ to $0.4 \mathrm{~Hz}) .{ }^{50}$ We applied amplitude thresholding method $^{50}$ either for $\Delta \mathrm{HbO}_{2}$ and $\Delta \mathrm{Hb}$ data in order to remove out motion artifacts. If amplitude exceeds threshold value for $\Delta \mathrm{Hb}(-0.5 \mu \mathrm{M})$ and $\Delta \mathrm{HbO}_{2}(0.5 \mu \mathrm{M})$, amplitude thresholding method was applied to related $\Delta \mathrm{HbO}_{2}$ and $\Delta \mathrm{Hb}$ channel.

\subsection{Statistical Analysis of Clinical Data}

Statistical analyses were performed with SPSS 20.0. We used Shapiro-Wilk test for normality of variables. We applied a $2 \times$ 2 (group $\times$ hand) ANOVA to compare pain thresholds between the groups. A $2 \times 2$ (group $\times$ condition) between/within subject design of ANOVA was applied for pain ratings. Group was between FM patients and HC and condition was within "pain + placebo TENS" and "pain + TENS." Statistics for pain ratings were run separately for each hand. Pearson correlation coefficient was used to associate fNIRS and behavioral data. To compare gender distribution, we used chi-square test. A $p$ value $\leq 0.05$ was considered for statistical significance.

\subsection{Brain Activity Data Analysis}

After preprocessing, we obtained mean $\Delta \mathrm{HbO}_{2}$ value for each condition. We performed the following analyses for each channel separately. In the first experiment, we ran $2 \times 2$ (group $\times$ hand) repeated measures ANOVA by using block averaged mean $\Delta \mathrm{HbO}_{2}$ of "TENS" condition. In the second experiment, we ran $2 \times 2$ (group $\times$ condition) repeated measures ANOVA using mean $\Delta \mathrm{HbO}_{2}$ values of "pain + placebo TENS" and "pain + TENS" conditions. For each channel, post hoc analyses were performed separately for significant factors/interactions by using Bonferroni correction similarly to the methodology used in several studies. ${ }^{20,54} \mathrm{~A} p$ value $\leq 0.05$ was considered for statistical significance.

\section{Results}

Demographic and clinical characteristics are shown in Table 2. Age and gender were similar between the groups (both $p>0.05)$. There was one postmenopausal woman in each group, 14 premenopausal women (eight in luteal and six in follicular phases) in $\mathrm{HC}$, and 16 premenopausal women (eight in luteal and eight in follicular phases) in FM patients. The BDI scores were higher in FM patients than $\mathrm{HC}[t(34): 3.304$, $p=0.004]$.

For pain thresholds, there were significant differences between FM patients and controls $[F(1,34): 7.788, \quad p=0.009]$, and between hands $[F(1,34): 5.467, p=0.025]$. Post hoc analysis showed that HC had higher pain threshold values than FM patients $(243.7 \pm 11.8$ versus $198.3 \pm 11.2$, mean difference; $45.4 \pm 16.3, p=0.009)$. In addition, the dominant hand had higher pain threshold than the nondominant hand $(226.9 \pm 8.5$ versus $215.2 \pm 8.6$, mean difference; $11.8 \pm 5.0, p=0.025$ ). Group and hand interaction showed a marginally significant difference between hands $[F(1,16): 3.817, \quad p=0.072]$, such that the pain threshold difference between hands of the FM patients differed significantly [ $t(18):-2.493, p=0.028]$.

During right-hand stimulation (RHS) with TENS, there was no significant subjective pain rating difference between the groups and conditions. Furthermore, there was no significant group and condition interaction. For pain ratings, left-hand stimulation (LHS) with TENS, there was no significant 
Table 2 Demographic and clinical characteristics of the participants. Data were given as mean \pm SD, ratio or median (min-max). FM, fibromyalgia, F, female; M, male; BDI, Beck Depression Inventory; FIQ, fibromyalgia impact questionnaire; gf, gram force; RHS, right-hand stimulation; and LHS, left-hand stimulation.

\begin{tabular}{|c|c|c|c|}
\hline Variable & FM patients $(N=19)$ & Healthy controls $(N=17)$ & Statistical results \\
\hline Age (years) & $37.7 \pm 5.8$ & $36.2 \pm 9.0$ & $p=0.537$ \\
\hline Gender (M/F) & $2 / 17$ & $2 / 15$ & $p=0.906$ \\
\hline BDI score & $19.6 \pm 10.1$ & $9.2 \pm 8.8$ & $p=0.004$ \\
\hline \multicolumn{4}{|l|}{ Pain threshold (gf) } \\
\hline Right thumb & $208.9 \pm 54.0$ & $244.8 \pm 46.8$ & Group: $p=0.009$ \\
\hline Left thumb & $183.3 \pm 56.7$ & $242.5 \pm 41.7$ & Hand: $p=0.025$ \\
\hline \multicolumn{4}{|l|}{ Pain ratings } \\
\hline RHS (pain + TENS) & $70.7 \pm 20.5$ & $64.3 \pm 25.3$ & Group (RHS); $p=0.581$ \\
\hline RHS (pain + placebo TENS) & $69.5 \pm 22.9$ & $67.3 \pm 24.9$ & Condition (RHS); $p=0.543$ \\
\hline LHS (pain + TENS) & $67.9 \pm 24.5$ & $64.8 \pm 28.6$ & Group (LHS); $p=0.748$ \\
\hline LHS (pain + placebo TENS) & $72.9 \pm 24.7$ & $70.2 \pm 28.4$ & Condition (LHS); $p=0.001$ \\
\hline Number of tender point & $14(11$ to 16$)$ & - & \\
\hline Disease duration (years) & $4.3 \pm 5.9$ & - & \\
\hline FIQ score & $61.3 \pm 13.9$ & - & \\
\hline
\end{tabular}

difference between groups, however, there was a significant difference between the conditions $[F(1,33)=13.14, p=0.001]$. There was no interaction between group and condition. Post hoc results indicated that, "pain + placebo TENS" condition ratings were higher than "pain + TENS" ratings (mean difference: $5.20 \pm 1.43$ ). This indicates that the relief effect caused by TENS was evident to all of the subjects for the left hand for both groups.

\subsection{Brain Activation Results: Quality Control}

Before statistical analysis, data from each channel and each participant were checked for quality control. One healthy subject was discarded due to no hemodynamic response based on correlation analysis with the applied task waveform. All results for median nerve stimulation are shown in Fig. 4, and for LHS and RHS of painful stimulation with TENS in Figs. 5 and 6,

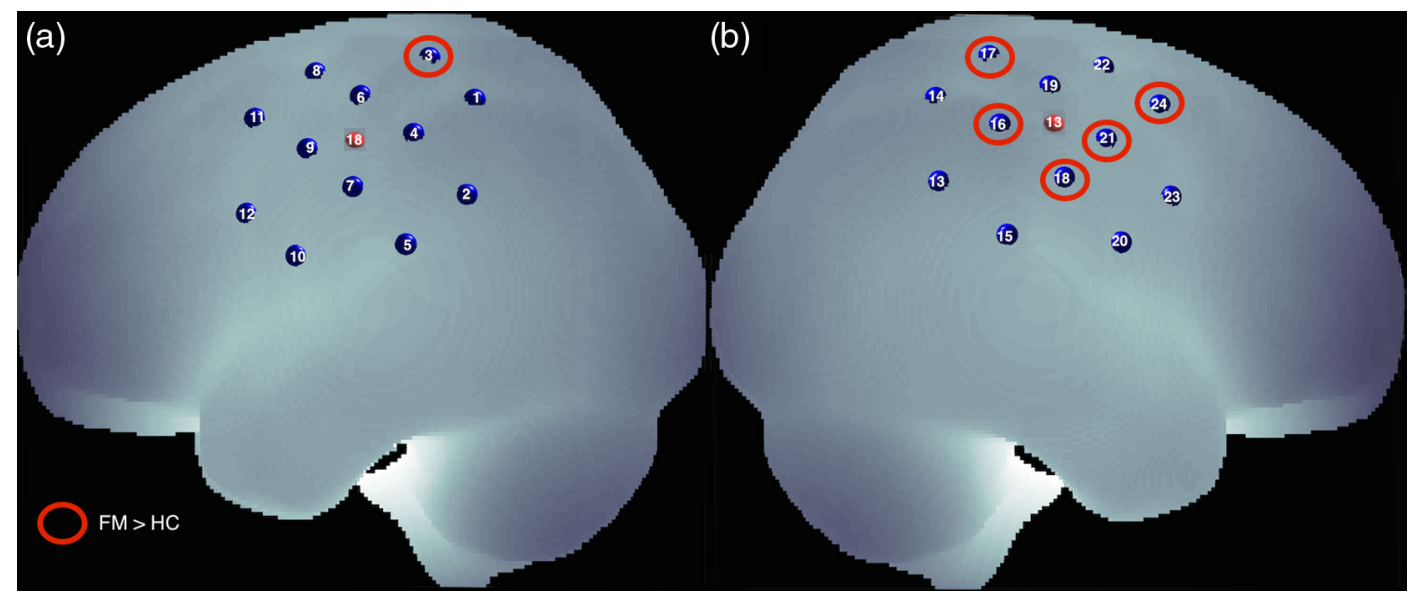

Fig. 4 Channels that show group main effect in median nerve stimulation with TENS marked on MNI space. (a) Left hemisphere and (b) right hemisphere ( $L$, left and $R$, right). Blue dots represent channels and red dots represent the sources which are considered as C3 and C4 in EEG 10-20 system (13 and 18). The difference found in group main effect was circled in red. FM, fibromyalgia patients, and HC, healthy controls. 


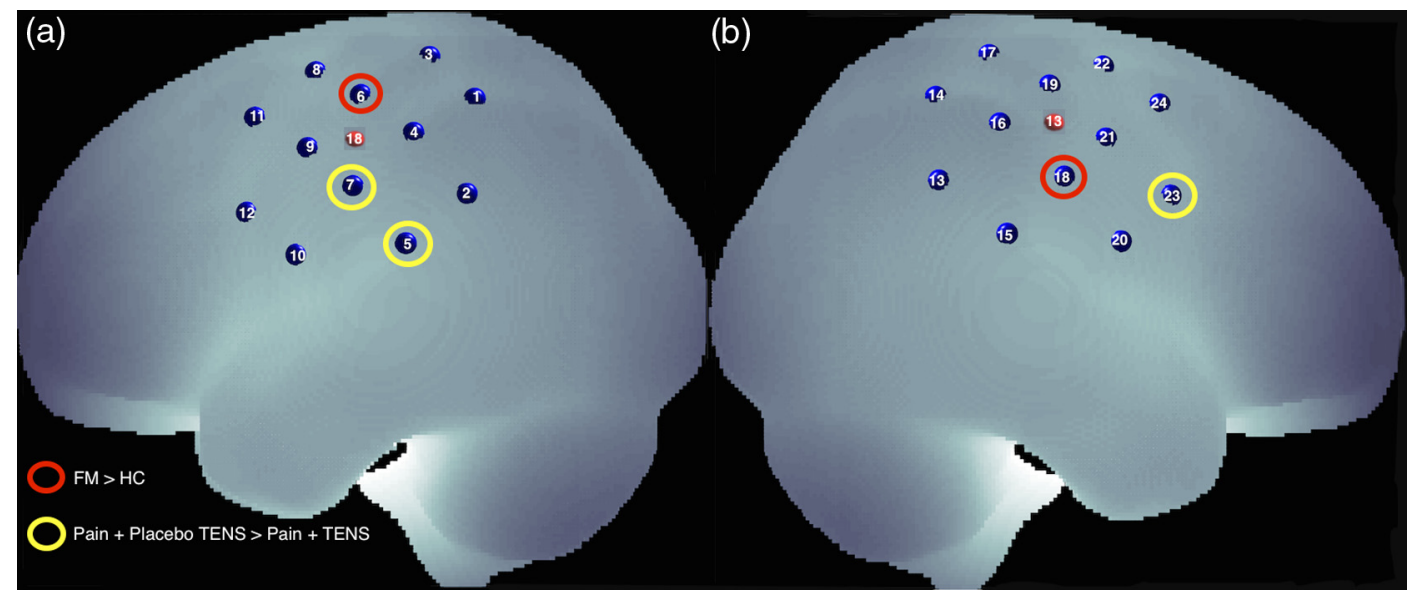

Fig. 5 Channels that show group and condition main difference for LHS. The difference found in group main effect and condition main effect was circled in red and yellow, respectively. (a) Left hemisphere and (b) right hemisphere. FM, fibromyalgia patients, and $\mathrm{HC}$, healthy controls.

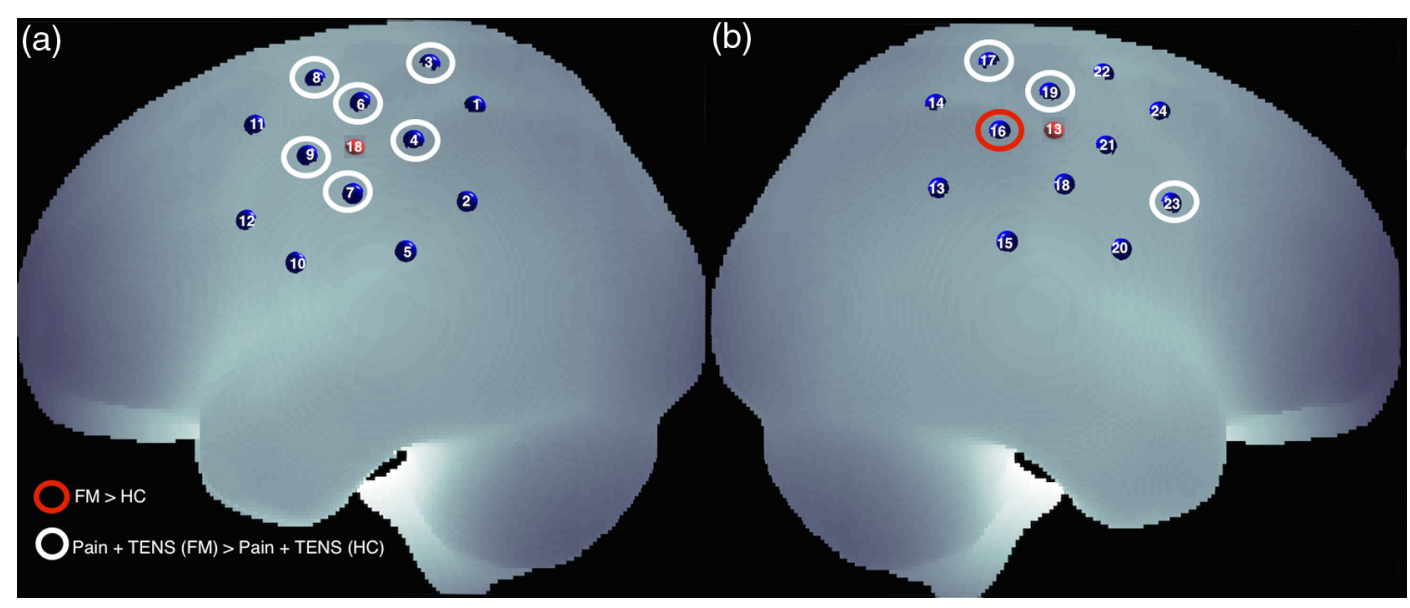

Fig. 6 Channels that show group main difference and interaction between group and condition for RHS. The difference found in group main effect and interaction between group and condition was circled in red and white, respectively. (a) Left hemisphere and (b) right hemisphere. FM, fibromyalgia patients, and $\mathrm{HC}$, healthy controls.

respectively. Anatomical locations and all statistical experimental results for all channels with their corresponding regions are shown in Table 3.

\subsubsection{Median nerve stimulation with TENS}

Results showed that there were significant group differences in left and right superior parietal gyrus (SPG) [channel 3; $F(1,33)=5.10, p=0.030$, channel $17 ; F(1,33)=7.37, p=$ $0.010]$, right supramarginal [channel $16 ; F(1,33)=5.92$, $p=0.021$, channel $18 ; F(1,33)=6.98, p=0.012]$, right SI [channel 21; $F(1,33)=5.77, p=0.022$ ], and right middle frontal gyrus (MFG) [channel 24; $F(1,33)=8.11$, $p=0.007]$. Pairwise comparison showed that FM patients showed higher activity than HC. None of these regions showed a significant difference in hand main effect or group $\times$ hand interaction. Channels that represent group effects are highlighted in Fig. 4. Time series of block average results of leftand right-hand TENS stimulations are shown in Appendix.

\subsubsection{Painful stimulation with TENS}

For LHS with TENS, significant differences were observed in both group and condition main effects (Fig. 5). Contralateral supramarginal [channel 18; $F(1,33)=4.54, p=0.041$ ] and ipsilateral SI [channel 6; $F(1,33)=6.75, p=0.014$ ] gyri showed a significant group main effect for the mean values. Post hoc analysis revealed that FM patients showed higher activity than $\mathrm{HC}$ in contralateral supramarginal gyrus (SMG) and ipsilateral SI. Condition main effect was observed in ipsilateral supramarginal [channel 5, $F(1,33)=9.52, p=0.004$ ], ipsilateral SI [channel $7, F(1,33)=8.54, p=0.006$ ], and contralateral precentral gyri (MI) [channel 23, $F(1,33)=4.47$, $p=0.042]$ gyri. Post hoc analysis revealed that "pain + placebo TENS" condition was found higher than "pain + TENS" condition in ipsilateral SMG and SI, and contralateral MI. No interaction between group and condition was found. These results reveal that the relieving effect of TENS was present for both FM and HC groups. Time series of block average results of LHS is shown in the Appendix A. 
Table 3 Results of median nerve stimulation with TENS and painful stimuli with TENS. Post hoc comparison results explained below the $p$ value. FM, fibromyalgia HC, healthy controls; n.s., not significant; LHS, left-hand stimulation; and RHS, right-hand stimulation.

\begin{tabular}{|c|c|c|c|c|c|c|c|c|c|c|}
\hline \multirow[b]{3}{*}{ Channel } & \multirow[b]{3}{*}{ Region } & \multirow{2}{*}{\multicolumn{3}{|c|}{$\begin{array}{l}\text { TENS experiment } \\
(P \text { vals, df: } 1,33)\end{array}$}} & \multicolumn{6}{|c|}{ Painful stimulation with TENS experiment (P vals, df: 1,33$)$} \\
\hline & & & & & \multicolumn{3}{|c|}{ LHS } & \multicolumn{3}{|c|}{ RHS } \\
\hline & & Group & Hand & $\begin{array}{l}\text { Group } \times \\
\text { hand }\end{array}$ & Group & Condition & $\begin{array}{l}\text { Group } \times \\
\text { condition }\end{array}$ & Group & Condition & Group $\times$ condition \\
\hline 1 & SPG & n.s & n.s & n.s & n.s & n.s & n.s & n.s. & n.s. & $\begin{array}{c}0.019 \\
\text { pain + TENS of } \\
\text { FM }>\text { pain + placebo } \\
\text { TENS of FM }\end{array}$ \\
\hline 2 & $A G$ & n.s & n.s & n.s & n.s & n.s & n.s & n.s. & n.s. & n.s. \\
\hline 3 & SPG & $\begin{array}{c}0.031 \\
\mathrm{FM}>\mathrm{HC}\end{array}$ & n.s & n.s & n.s & n.s & n.s & n.s. & n.s. & $\begin{array}{c}0.009 \\
\text { pain }+ \text { TENS of } \\
\text { FM }>\text { pain + placebo } \\
\text { TENS of FM }\end{array}$ \\
\hline 4 & SMG & n.s & n.s & n.s & n.s & n.s & n.s & n.s. & n.s. & $\begin{array}{c}0.016 \\
\text { pain }+ \text { TENS of } \\
\text { FM }>\text { pain }+ \text { placebo } \\
\text { TENS of FM }\end{array}$ \\
\hline 5 & SMG & n.s & n.s & n.s & n.s & $\begin{array}{c}0.004 \\
\text { pain + placebo } \\
\text { TENS > pain + } \\
\text { TENS }\end{array}$ & n.s & n.s. & n.s. & n.s. \\
\hline 6 & Post-CG & n.s & n.s & n.s & $\begin{array}{c}0.014 \\
F M>~ H C\end{array}$ & n.s & n.s & n.s & n.s. & $\begin{array}{c}0.011 \\
\text { pain + TENS of } \\
\text { FM > pain + placebo } \\
\text { TENS of FM }\end{array}$ \\
\hline 7 & Post-CG & n.s & n.s & n.s & n.s & $\begin{array}{c}0.006 \\
\text { pain + placebo } \\
\text { TENS > pain + } \\
\text { TENS }\end{array}$ & n.s & n.s. & n.s. & $\begin{array}{c}0.036 \\
\text { pain + TENS of } \\
\text { FM }>\text { pain + placebo } \\
\text { TENS of FM }\end{array}$ \\
\hline 8 & Pre-CG & n.s & n.s & n.s & n.s & n.s & n.s & n.s. & n.s. & $\begin{array}{c}0.010 \\
\text { pain + TENS of } \\
\text { FM > pain + placebo } \\
\text { TENS of FM }\end{array}$ \\
\hline 9 & Pre-CG & n.s & n.s & n.s & n.s & n.s & n.s & n.s. & n.s. & $\begin{array}{c}0.037 \\
\text { pain + TENS of } \\
\text { FM }>\text { pain + placebo } \\
\text { TENS of FM }\end{array}$ \\
\hline 10 & Post-CG & n.s & n.s & n.s & n.s & n.s & n.s & n.s. & n.s. & n.s. \\
\hline 11 & MFG & n.s & n.s & n.s & n.s & n.s & n.s & n.s. & n.s. & n.s. \\
\hline 12 & Pre-CG & n.s & n.s & n.s & n.s & n.s & n.s & n.s. & n.s. & n.s. \\
\hline 13 & $A G$ & n.s & n.s & n.s & n.s & n.s & n.s & n.s. & n.s. & $\begin{array}{c}0.023 \\
\text { pain + TENS of } \\
\text { FM > pain + placebo } \\
\text { TENS of FM }\end{array}$ \\
\hline 14 & SPG & n.s & n.s & n.s & n.s & n.s & n.s & n.s. & n.s. & $\begin{array}{c}0.005 \\
\text { pain }+ \text { TENS of } \\
\text { FM }>\text { pain }+ \text { placebo } \\
\text { TENS of FM }\end{array}$ \\
\hline 15 & SMG & n.s & n.s & n.s & n.s & n.s & n.s & n.s. & n.s. & n.s. \\
\hline
\end{tabular}


Table 3 (Continued).

\begin{tabular}{|c|c|c|c|c|c|c|c|c|c|c|}
\hline \multirow[b]{3}{*}{ Channel } & \multirow[b]{3}{*}{ Region } & \multirow{2}{*}{\multicolumn{3}{|c|}{$\begin{array}{l}\text { TENS experiment } \\
\text { ( } P \text { vals, df: } 1,33 \text { ) }\end{array}$}} & \multicolumn{6}{|c|}{ Painful stimulation with TENS experiment ( $P$ vals, df: 1,33$)$} \\
\hline & & & & & \multicolumn{3}{|c|}{ LHS } & \multicolumn{3}{|c|}{ RHS } \\
\hline & & Group & Hand & $\begin{array}{l}\text { Group } \times \\
\text { hand }\end{array}$ & Group & Condition & $\begin{array}{l}\text { Group } \times \\
\text { condition }\end{array}$ & Group & Condition & Group $\times$ condition \\
\hline 16 & SMG & $\begin{array}{c}0.021 \\
\mathrm{FM}>\mathrm{HC}\end{array}$ & n.s & n.s & n.s & n.s & n.s & $\begin{array}{c}0.044 \\
\mathrm{FM}>\mathrm{HC}\end{array}$ & n.s. & n.s. \\
\hline 17 & SPG & $\begin{array}{c}0.010 \\
F M>H C\end{array}$ & n.s & n.s & n.s & n.s & n.s & n.s. & n.s. & $\begin{array}{c}0.003 \\
\text { pain }+ \text { TENS of } \\
\text { FM }>\text { pain }+ \text { placebo } \\
\text { TENS of FM }\end{array}$ \\
\hline 18 & SMG & $\begin{array}{c}0.012 \\
F M>H C\end{array}$ & n.s & n.s & $\begin{array}{c}0.041 \\
F M>H C\end{array}$ & n.s & n.s & n.s. & n.s. & n.s. \\
\hline 19 & Post-CG & n.s & n.s & n.s & n.s & n.s & n.s & n.s. & n.s. & $\begin{array}{c}0.038 \\
\text { pain + TENS of } \\
\text { FM }>\text { pain + placebo } \\
\text { TENS of FM }\end{array}$ \\
\hline 20 & Post-CG & n.s & n.s & n.s & n.s & n.s & n.s & n.s. & n.s. & $\begin{array}{c}0.048 \\
\text { pain }+ \text { TENS of } \\
\text { FM > pain + placebo } \\
\text { TENS of FM }\end{array}$ \\
\hline 21 & Post-CG & $\begin{array}{c}0.022 \\
\mathrm{FM}>\mathrm{HC}\end{array}$ & n.s & n.s & n.s & n.s & n.s & n.s & n.s. & n.s. \\
\hline 22 & Pre-CG & n.s & n.s & n.s & n.s & n.s & n.s & n.s. & n.s. & $\begin{array}{c}0.025 \\
\text { pain + TENS of } \\
\text { FM }>\text { pain + placebo } \\
\text { TENS of FM }\end{array}$ \\
\hline 23 & Pre-CG & n.s & n.s & n.s & n.s & $\begin{array}{c}0.042 \\
\text { pain + placebo } \\
\text { TENS > pain + } \\
\text { TENS }\end{array}$ & n.s & n.s. & n.s. & $\begin{array}{c}0.037 \\
\text { pain + TENS of } \\
\text { FM > pain + placebo } \\
\text { TENS of FM }\end{array}$ \\
\hline 24 & MFG & $\begin{array}{c}0.007 \\
\mathrm{FM}>\mathrm{HC}\end{array}$ & n.s & n.s & n.s & n.s & n.s & n.s. & n.s. & n.s. \\
\hline
\end{tabular}

For RHS with TENS, during painful stimulus administration, the main effect between the groups was observed in ipsilateral SMG [channel 16; $F(1,33)=4.39, p=0.044$ ], as shown in Fig. 6. Pairwise comparison showed that cortical activity was greater in FM patients than controls in SMG. For condition main effect, there was no difference between "pain + placebo TENS" and "pain + TENS" conditions in any channel.

Widespread bilateral significant interactions were observed for RHS, but there was no significant group and condition interaction in LHS. For RHS, seven channels in the right hemisphere and six channels in the left hemisphere revealed significant interactions in brain activity. It was found that this interaction was mainly due to FM patients having more brain activity for the "pain + TENS" condition compared to the "pain + placebo TENS" condition, whereas healthy participants exhibited a reversed pattern (i.e., more brain activity for the "pain + placebo TENS" condition compared to the "pain + TENS" condition).

Interactions were found in the bilateral SPG [channel 1; $F(1,33)=6.13, \quad p=0.019$, channel $3 ; \quad F(1,33)=7.70$, $p=0.009$, channel $14 ; F(1,33)=8.88, p=0.005$, channel $17 ; F(1,33)=10.35, p=0.003$ ], bilateral SI [channel 6; $F(1,33)=7.33, \quad p=0.011 ;$ channel $7 ; \quad F(1,33)=4.78$, $p=0.036$; channel $19 ; F(1,33)=4.65, p=0.038$, channel $20 ; F(1,33)=4.21, p=0.048$ ], bilateral MI [channel 8; $F(1,33)=7.53, \quad p=0.010 ;$ channel $9 ; \quad F(1,33)=4.74$, $p=0.037$; channel $22 ; F(1,33)=5.78, p=0.025$, channel $23 ; F(1,33)=4.74, \quad p=0.037]$, ipsilateral angular gyrus (AG) [channel $13 ; F(1,33)=5.71, p=0.022$ ], and contralateral SMG [channel $4 ; F(1,33)=6.40, p=0.016$ ] for RHS. In channels $1,3,4,6,7,8,9,13,14,17,19,20,22$, and 23, post hoc analyses revealed that "pain + TENS" condition caused higher activation compared with "pain + placebo TENS" condition in FM patients. Time series of block average results of RHS is shown in Appendix.

When results from both experiments are merged for RHS for channels 3 and 17 (left and right SPGs), a meaningful contribution of TENS emerges. Interaction between groups (FM and controls) and conditions ("pain + placebo TENS" and "pain + 

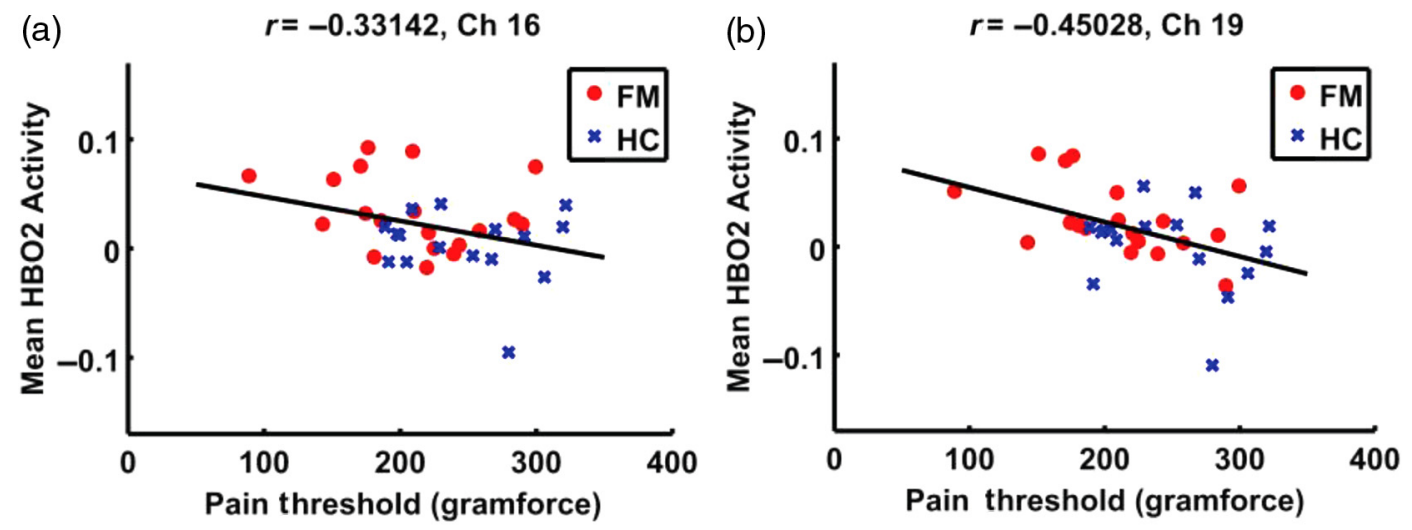

Fig. 7 Correlation plots of (a) right-hand pain threshold and block averaged mean $\Delta \mathrm{HbO}_{2}$ of right-hand stimulation of "pain + TENS" for channel 16 and (b) right-hand pain threshold and block averaged mean $\Delta \mathrm{HbO}_{2}$ of right-hand stimulation of "pain + TENS" for channel 19.

TENS") was due to the hyper sensitivity of FM patients to TENS. In no other channel, this type of brain activity pattern was revealed.

\subsection{Correlations Between fNIRS and Behavioral Data}

In RHS, among these channels, significant negative correlations were observed between dominant hand pain threshold and dominant hand mean $\Delta \mathrm{HbO}_{2}$ of "pain + TENS" condition in the ipsilateral supramarginal (channel $16 ; r=-0.33, p=0.05$ ) and between dominant hand mean $\Delta \mathrm{HbO}_{2}$ of "pain + TENS" condition in the ipsilateral SI (channel 19; $r=-0.45$, $p=0.006)$ gyri. This indicates that the subjects with a higher pain threshold had lower mean brain activity in the pain + TENS condition compared to the other subjects. All these correlation results are shown in Fig. 7.

For LHS, we determined to focus on channels that had group or condition main effect (channels 5, 6, 7, 18, and 23). Among these channels, there was no significant correlation between pain ratings and pain thresholds.

\section{Discussion}

In this study, we investigated the effects of TENS in both FM patients and $\mathrm{HC}$ during noxious stimulation. Based on earlier literature in gate control theory, we hypothesized that hemodynamic activity would decrease when TENS stimulation was applied along with nociceptive stimuli in comparison to the "pain + placebo TENS" condition. TENS is used to control acute/chronic pain; however, its mechanism depends on some parameters, i.e., frequency, intensity, and amplitude. Conventional TENS used in this study would act in peripherally through the gate control theory and thus, has an analgesic effect during application. We demonstrated that painful stimuli together with TENS application to the nondominant side resulted in similar TENS effects (decreased nociceptive inputs peripherally and blocks the arrival of painful sensation in the central nervous system) in both healthy subjects and FM patients. On the other hand, while painful stimulus and TENS applied to the dominant side resulted in decreased brain activity in controls, an increased activation was observed in FM patients. This finding indicates that TENS can cause allodynia possibly due to peripheral sensitization and central amplification when the dominant side is stimulated. Thus, we can say that the stimulation side might be an important factor in the treatment of FM while using TENS.

We performed median nerve stimulation with TENS to observe the baseline effects of TENS. For everyone, a stimulation intensity of about $30 \mathrm{~mA}$ was applied so that a nonnociceptive tingling effect was delivered. Such low-intensity and highfrequency application is called "conventional TENS", 55 which is used for pain relief. In this experiment, we observed group difference in left and right SPGs, right SMG, right SI, and right MFG. Post hoc analysis revealed that FM patients showed higher brain activation than HC for TENS stimulation. This is a replication of another nonpainful stimulation study with a thermal stimulator. ${ }^{28}$ Cook et al. ${ }^{28}$ showed that FM patients exhibited greater activity than controls in prefrontal, supplementary motor area (SMA), insular, and ACC. Central nervous system dysregulation was found independent of stimulus type in FM patients. ${ }^{28}$ For instance, when visual, auditory, and tactile (finger tapping) stimulation were used, higher activation in FM patients than controls was observed in insula and lingual gyrus. ${ }^{36}$ Increased activation in FM patients is a general pattern, which indicates that TENS related activity in FM patients might increase due to central sensitization. Because stimulated large $\mathrm{A} \beta$ nerve fibers that carries nonnociceptive stimulation causes allodynia in FM patients. ${ }^{41}$ Allodynia is the increased response of neurons to nonpainful stimulation such as light touch. It is related to nerve damage. ${ }^{56} \mathrm{FM}$ patients may consider TENS stimulation as a nociceptive stimulation which in turn introduces additive effect to hemodynamic activation. Lopez-Sola et al. ${ }^{36}$ explained this condition as a possible part of pathology in FM.

In the second experiment, we delivered nociceptive stimulus based on individual pain thresholds of each subject. During delivery of nociceptive stimulus, there were two conditions; "pain + placebo TENS" and "pain + TENS." In the literature, application of TENS reduced hemodynamic activity during painful stimulation for $\mathrm{HC}^{22}$ We expected a similar result for FM group as well by applying painful stimulation to both hands of FM and control groups. We investigated the pain relief effect of TENS. In the following, we present findings in detail for LHS and RHS separately.

\subsection{Left (Nondominant) Hand Stimulation}

LHS data indicated that significant differences were found in only group and condition main effects. We observed group 
main effect in contralateral SMG and ipsilateral SI. Post hoc analysis revealed that FM patients showed higher brain activity than HC. On the other hand, condition main effect was observed in ipsilateral SI and SMG and contralateral MI. Post hoc analysis showed that "pain + TENS" condition showed decreased hemodynamic activity compared to "pain + placebo TENS" condition in these channels. These findings reflect that TENS does have an effect to reduce somatosensory brain activity in both FM and healthy groups. In support of our findings, recent studies on FM patients also demonstrated that TENS causes a significant pain reduction. ${ }^{1,4,5,7}$ Activity reduction via TENS was found in right SMG located in IPL. Such a reduction in IPL was also reported in a recent study performed on healthy participants. $^{22}$

The TENS effect we observed in ipsilateral SMG (BA40) located in IPL is a replication with a previous fMRI study that showed the TENS effect in IPL while applying nociceptive stimuli in controls. ${ }^{22}$ IPL was found active when nociceptive stimulus was applied both in controls and FM patients. 27,30,33 However, the roots of pain perception with respect to IPL have not been clarified. IPL has an important role in attention to the noxious stimuli. Decrease of hemodynamic activity in "pain + TENS" condition might be related to a decrease in hypervigilance to nociceptive stimuli.

The LHS pain ratings showed that groups sensed nociceptive stimulation similarly because there was no difference in pain ratings. However, significant difference was found in condition main effect of the pain ratings. Post hoc analysis revealed that TENS efficiency was perceived by subjects in subjective pain ratings. This behavioral finding supports our condition main effect in hemodynamic activity results. We conclude that for the nondominant hand, TENS efficiency can be represented both in HC and FM patients in parietal and somatosensory cortices, which have important roles in pain perception.

\subsection{Right (Dominant) Hand Stimulation}

For RHS, when compared with HC, increased hemodynamic activity was found in FM group in the ipsilateral SMG, which is one of the two main components of IPL. Several studies showed that in this area, higher hemodynamic activities were observed in FM patients compared to HC. ${ }^{27-29,33,57}$ This might be related with hypersensitivity to nociceptive stimuli. Furthermore, IPL was found active in pain processing when equal pain sensation experiment was conducted. ${ }^{27,33}$ Activity differences of both groups in the ipsilateral SMG might be related to augmented pain processing among patients in this area.

On the other hand, we could not observe any difference for the condition main effect for RHS. This was obviously due to the significant interactions observed between group and condition in bilateral SI, MI, and SPG, and contralateral SMG. Post hoc results revealed that "pain + TENS" activity in FM patients was higher than the "pain + placebo TENS" condition, whereas in HC, a reverse pattern was observed. Several studies showed that brain activity to nociceptive stimulation was higher in FM patients than controls in SI, IPL (including AG), ${ }^{27,33}$ and MI. ${ }^{33}$ As superior parietal lobe is classified as sensory association cortex, an increase in hemodynamic activation in FM patients in this region is noteworthy.

In FM patients, contradictory to the decrease we expected with the use of TENS, the increase in hemodynamic response might reflect a malfunction of the gate control mechanism.
Woolf et al. suggested that nonnociceptive stimulation causes allodynia and secondary hyperalgesia that might occur due to peripheral sensitization in individuals with central sensitization. ${ }^{41}$ According to Cook et al., ${ }^{28}$ central nervous system dysregulation was found independent of stimulus type in FM patients. FM patients may consider TENS as a nociceptive stimulation, which may have introduced an increase in hemodynamic activation. Such alteration in pain processing mechanisms due to changes in peripheral and central nervous systems was reported previously. ${ }^{58}$ Increased activations of FM patients in "pain + TENS" condition within bilateral MI might also be due to attentional mechanisms triggered by TENS. However, this reasoning is somehow speculative as pain ratings did not change between the two conditions. On the other hand, the negative correlation between hemodynamic activity obtained by RHS and right-hand pain threshold in supramarginal and SPG indicate a clear association between the lower pain thresholds and higher "pain + TENS" activity. In addition, a negative correlation between "pain + placebo TENS" condition ratings and block averaged mean $\Delta c_{\mathrm{HBO} 2}$ was observed in left SPG (channel 3). In a recent study, negative correlation between painful stimulation and pain ratings was observed in FM patients before painful stimulation and subsequent pain in MI, SMA, and midcingulate cortex. ${ }^{34}$ Our finding in SPG by using both groups (FM and $\mathrm{HC}$ ) is complementary to this finding.

When results from the two experiments are merged for the RHS, we encountered a very important finding in bilateral SPG (channels 3 and 17). In these regions, while TENS activity in FM patients was found higher than $\mathrm{HC}$ in the median nerve stimulation experiment, RHS experiment showed that "pain + TENS" activity was found higher in FM patients than HC. Therefore, this activity increase in FM patients compared to HC during painful stimulation with TENS seems to be related to hypersensitivity of FM patients to TENS. Perhaps, this can be explained with allodynia in FM patients. On the contrary, the activity decrease in $\mathrm{HC}$ during painful stimulation with TENS compared to painful stimulation alone is an expected result, which can be explained the "gate control theory of pain." Such a striking difference between groups is not present in the LHS experiments we performed. There is not enough knowledge in FM literature that explains such a dichotomy between the pain processing in right versus left hands.

Although hand dominancy is an active research area in pain perception, there is no common agreement about the effect of hand dominancy in this domain. Some studies show that pain perception is different between hands, ${ }^{59-62}$ but others do not agree about this difference. ${ }^{63-66}$ However, if there is a difference between hands, nondominant hand shows a higher sensitivity to nociceptive stimulus. ${ }^{67-73}$ This conflict shows that while analyzing pain perception on either psychophysical studies or neuroimaging studies, hand dominancy effect should be considered as an effective factor. Moreover, some studies showed that pain threshold measured by a pressure algometer is greater in dominant hand than nondominant hand of healthy participants. ${ }^{59,60,68-71,38}$

On the other hand, there are several neuroimaging studies that include painful stimulation to both hands. ${ }^{23-26}$ These studies were performed by using noxious laser, ${ }^{23,24}$ electrical stimulation, ${ }^{25}$ and heat stimulation. ${ }^{26}$ These studies showed that there is bilateral activation and contralateral bias in SI, SII, insula, and thalamus ${ }^{24,26}$ and also motor output related structures such as putamen and cerebellum. ${ }^{23}$ Also, these studies suggested 
Eken et al.: Differential efficiency of transcutaneous electrical nerve stimulation in dominant versus...

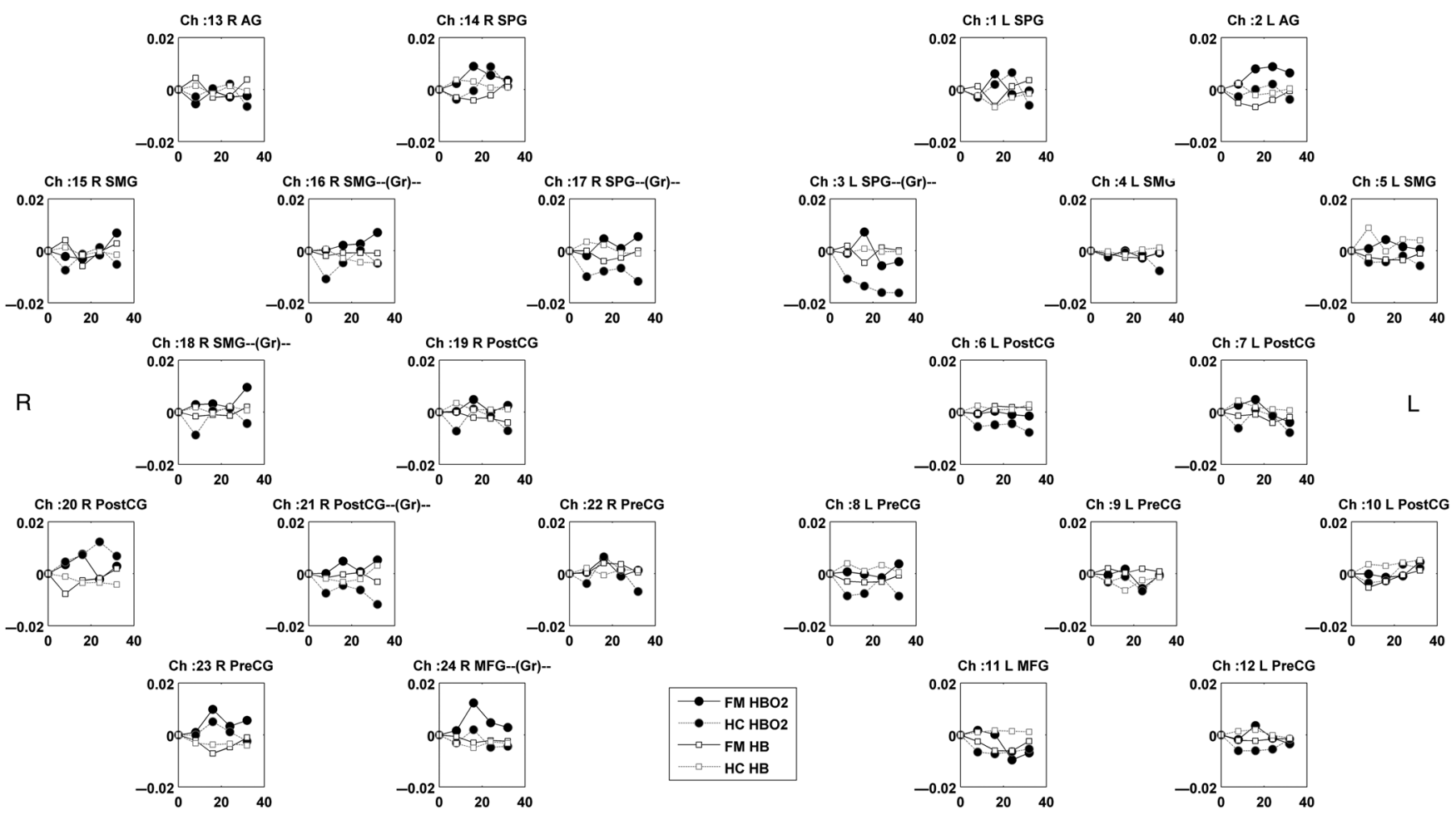

Fig. 8 Block average time series results of left-hand TENS stimulation. R, right; L, left; Gr, channels that show group difference in $2 \times 2$ (group $\times$ hand) repeated measures ANOVA results; AG, angular gyrus; SPG, superior parietal gyrus; SMG, supramarginal gyrus; pre-CG, precentral gyrus; post-CG, postcentral gyrus; MFG, middle frontal gyrus; $\mathrm{FM}$, fibromyalgia; $\mathrm{HC}$, healthy controls; $\mathrm{HBO}_{2}$, oxy-hemoglobin activity; and $\mathrm{HB}$, deoxy-hemoglobin activity.
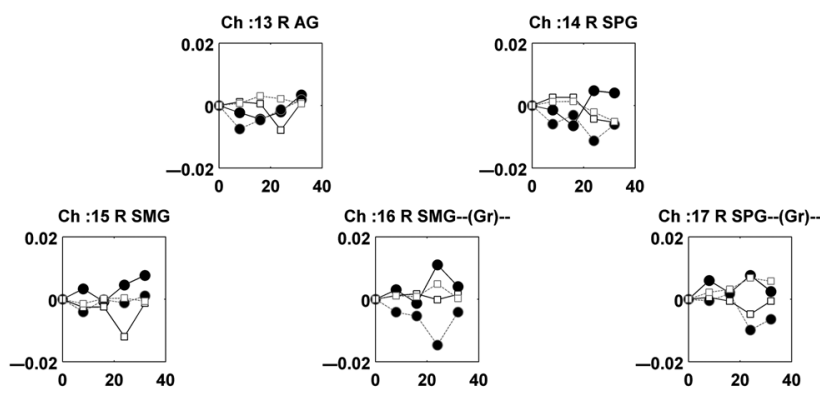

$\mathrm{R}$

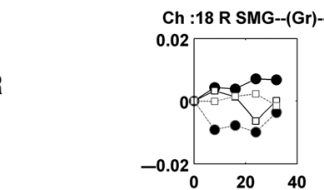

Ch:19 R PostcG
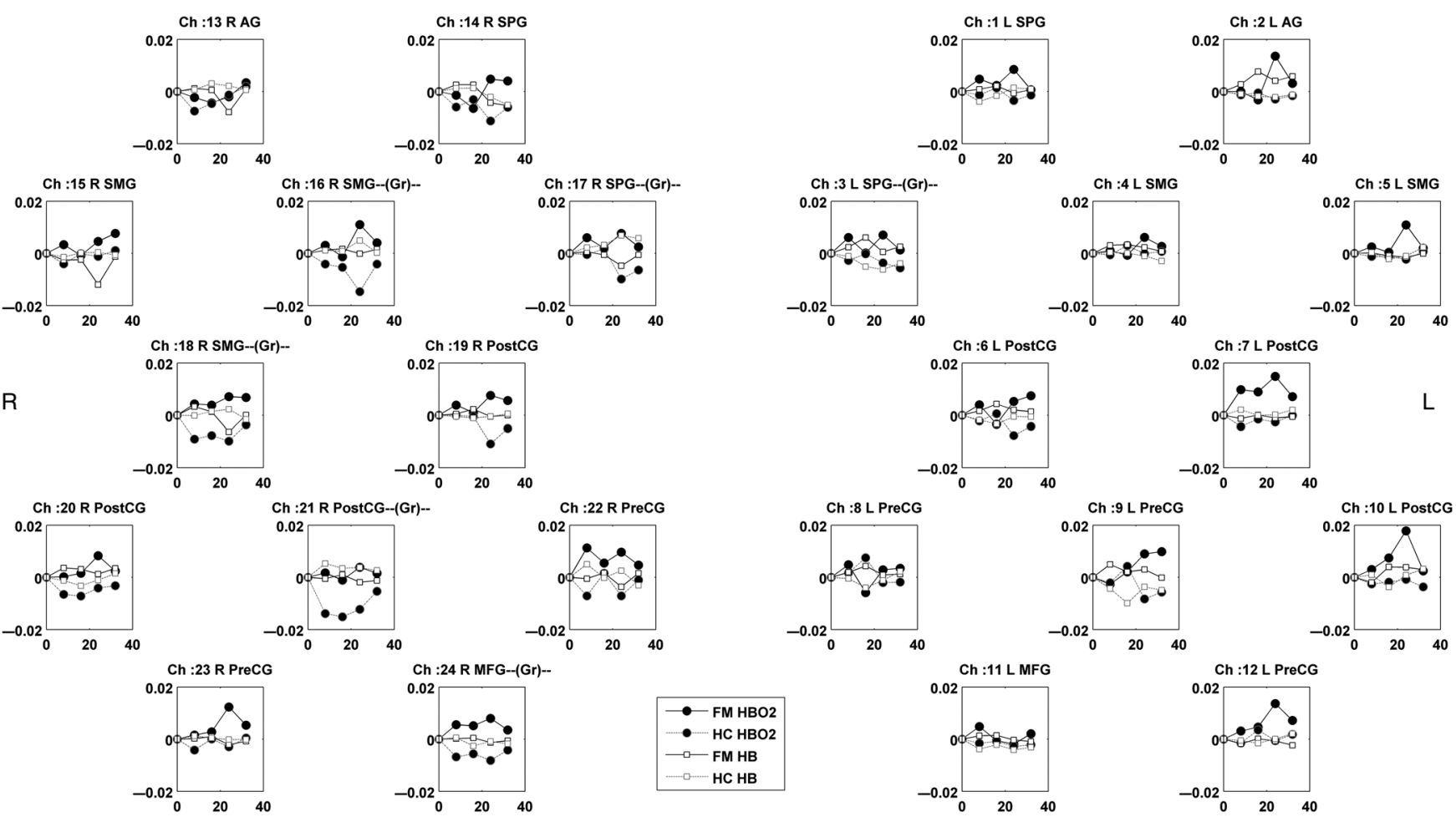

Fig. 9 Block average time series results of right-hand TENS stimulation. R, right; L, left; Gr, channels that show group difference in $2 \times 2$ (group $\times$ hand) repeated measures ANOVA results; AG, angular gyrus; SPG, superior parietal gyrus; SMG, supramarginal gyrus; pre-CG, precentral gyrus; post-CG, postcentral gyrus; and MFG, middle frontal gyrus. 
Eken et al.: Differential efficiency of transcutaneous electrical nerve stimulation in dominant versus...

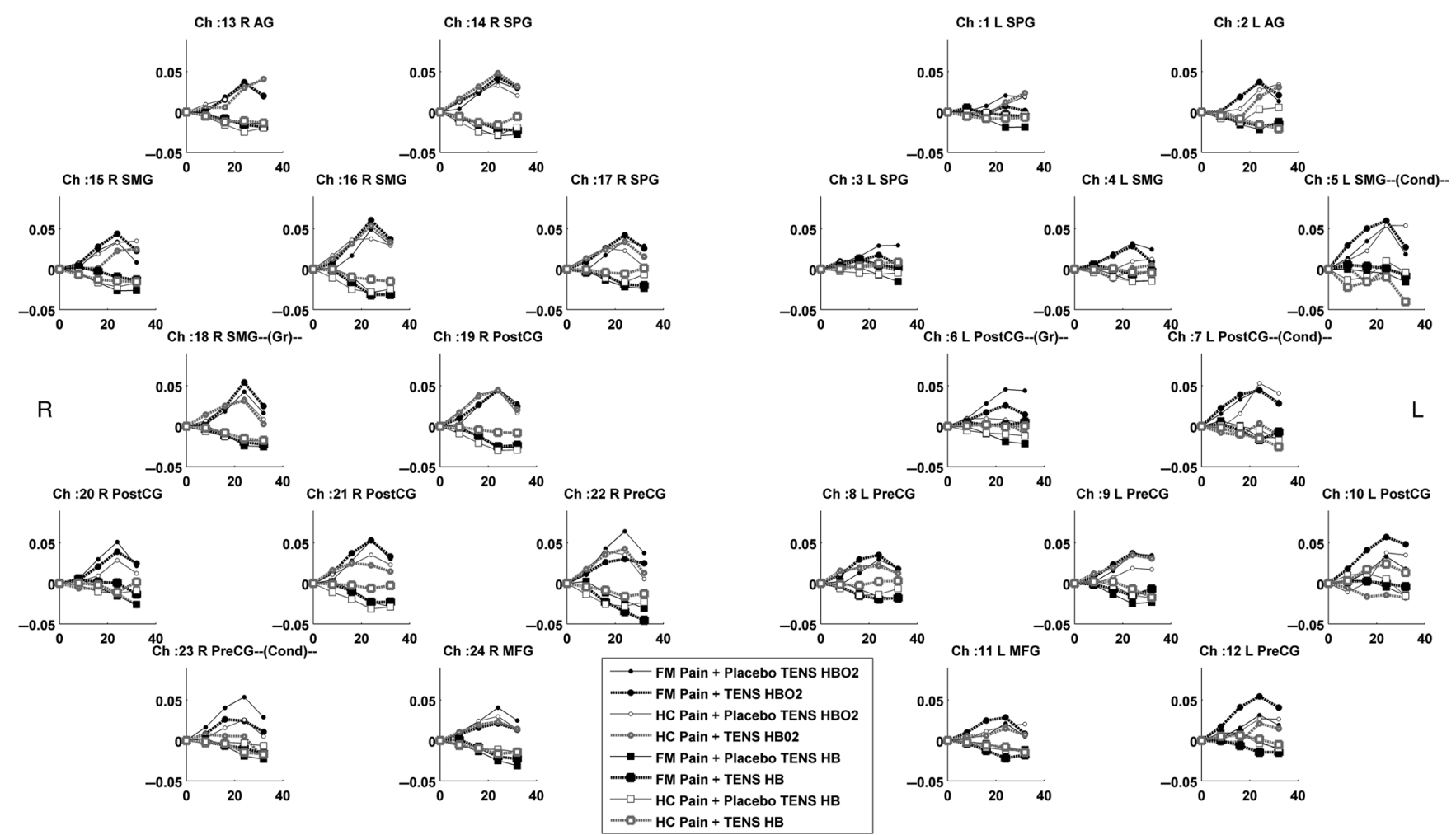

Fig. 10 Block average time series results of LHS. R, right and L, left. For $2 \times 2$ (group $\times$ condition) repeated measures ANOVA for LHS. Gr, channels that show significant group difference; Cond, channels that show significant condition difference; AG, angular gyrus; SPG, superior parietal gyrus; SMG, supramarginal gyrus; pre-CG, precentral gyrus; post-CG, postcentral gyrus; and MFG, middle frontal gyrus.

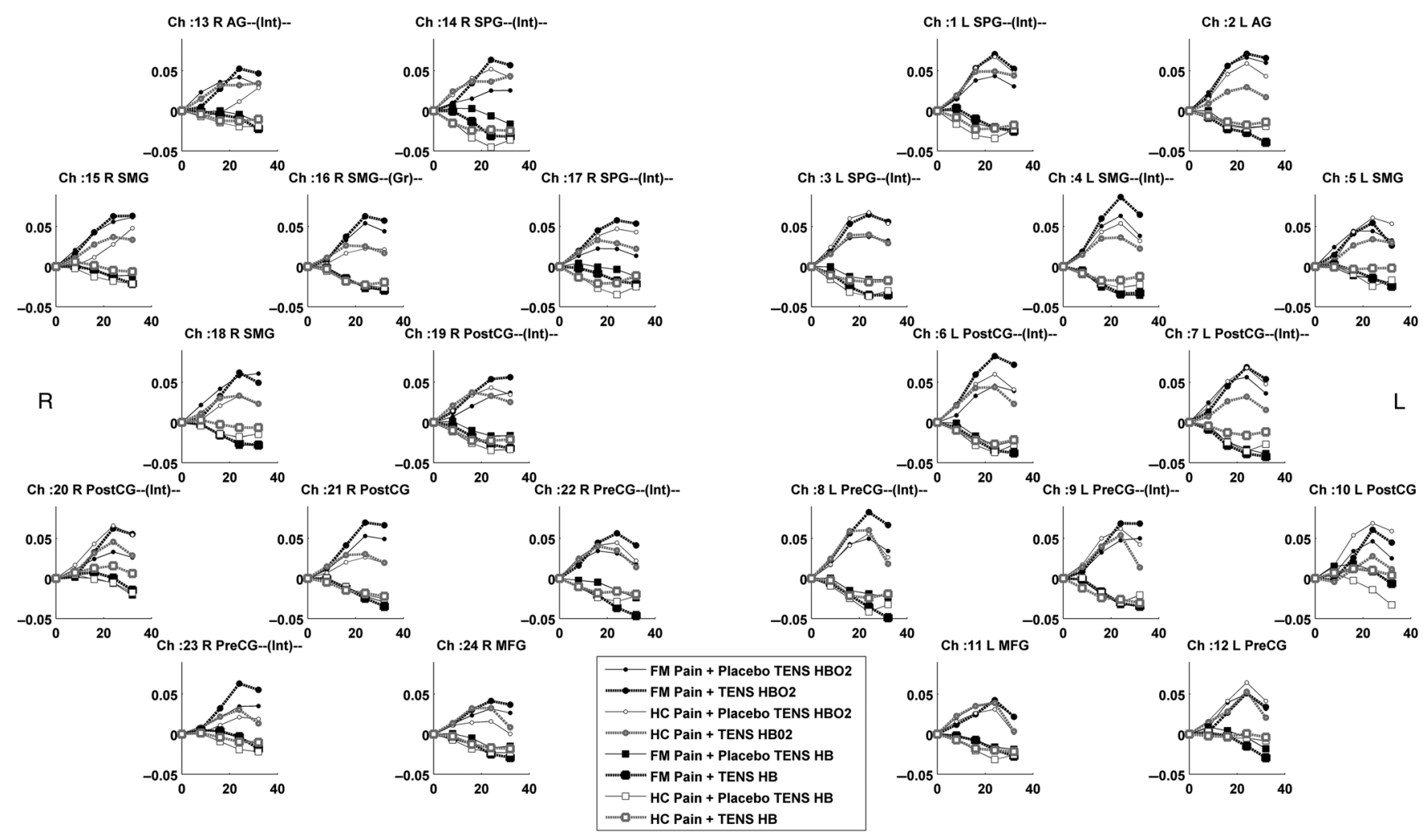

Fig. 11 Block average time series results of RHS. R, right and L, left. For $2 \times 2$ (group $\times$ condition) repeated measures ANOVA for RHS. Gr, channels that show significant group difference; Int, channels that show significant group and condition interaction; $A G$, angular gyrus; SPG, superior parietal gyrus; SMG, supramarginal gyrus; pre-CG, precentral gyrus; post-CG, postcentral gyrus; and MFG, middle frontal gyrus. 
that pain processing was strongly lateralized to the right hemisphere especially in MFG, ACC, inferior frontal gyrus, medial/ superior frontal gyrus, and IPL. ${ }^{25}$ Furthermore, a meta-analysis was performed by using activation likelihood estimate method. ${ }^{74}$ In this meta-analysis, hemispheric lateralization of pain perception was analyzed by comparing two groups of studies on rightand left-hand stimulations. Results showed that without considering the left or right stimulation, insular cortex and right ACC showed the most significant probabilistic values, which are generally observed in several pain studies. ${ }^{13}$ For LHS, the likelihood of activation was found significant in right SI, MI, posterior parietal cortex, and superior frontal gyrus, and for RHS, left SI, ACC, MI, IPL, and MFG. The likelihood of activation in ipsilateral side was found significant in midbrain for LHS. For RHS, ACC, IPL, and MFG showed the significant likelihood activation.

A recent study conducted a similar study to ours (on feet rather than hands) in healthy participants reported an increase in "pain + TENS "activity compared to "pain only" condition in SI. ${ }^{22}$ This would have meant that dominant-side effect of TENS is similar in both patient and healthy groups, but our finding in the healthy group is reverse. A recent study in healthy subjects showed that TENS efficiency on pain site might be related to stimulation intensity. ${ }^{75}$ While Choi et al. ${ }^{22}$ applied a mean of 16-mA TENS intensity, we applied about $30 \mathrm{~mA}$. Hence, stimulus intensity might be a factor just as important as the stimulated side.

The RHS findings showed that a subjectively adjusted amount of stimulation based on pain thresholds caused similar pain ratings for both groups consistent with the previous studies. ${ }^{27,33}$ Surprisingly, we found no difference between the ratings of the conditions. According to our results, none of the groups indicated differential pain perception during the "pain + TENS" condition, compared to the "pain + placebo TENS" condition. This indicates that the brain activity reduction in $\mathrm{HC}$ as well as brain activity increase in FM patients during "pain + TENS" condition was not carried on to conscious levels. Further studies are needed to isolate the underlying factors for this phenomenon. On the contrary, based on RHS mean $\mathrm{HBO}_{2}$ activity findings, there was a negative and moderate correlation between pain thresholds and brain activity, which indicates that increasing pain thresholds produce less brain activity showing pain processing efficiency [Figs. 7(a) and 7(b)].

\subsection{Limitations}

While performing statistical analyses, we assumed that the channels are independent. This means that any probable cross-talk between the channels was disregarded. The perceived pain ratings between the conditions were not significant. Therefore, the neuroimaging findings presented here only indicate unconscious aspects of pain. Our findings are generalizable insofar as female patients are of concern.

\section{Conclusion}

In this study, we investigated the effects of TENS both in FM patients and controls. This is the first fNIRS study investigating TENS effect during nociceptive stimulation with stimulation side in FM patients. According to our findings, reduction in hemodynamic activity due to TENS application was observed in $\mathrm{HC}$ for both sides. However, the effects of TENS differed with respect to the stimulation side of FM patients. While TENS caused similar brain activity reductions in FM patients and controls for the nondominant hand, it caused an increase in hemodynamic activity for the dominant hand in FM patients. These findings indicate that when TENS is applied to the dominant side, central sensitization-rather than gate control theory-might be more explanatory regarding the role of nonnociceptive interventions in FM. In the clinic, TENS treatment might not be as effective as expected on the dominant side of FM patients. In this sense, caution should remain as the prerequisite when treating lateralized painful conditions on the dominant side. Although FM is normally a widespread chronic painful condition, our findings indicate that asymmetric involvement or accompanying painful conditions may well ensue in FM patients.

\section{Appendix}

Time series of left- and right-hand stimulations of TENS are shown in Figs. 8 and 9 and time series of LHS and RHS are shown in Figs. 10 and 11. In these figures, channels were aligned and represented as it is shown in channel configuration.

\section{Disclosures}

The authors declare that there are no conflicts of interest regarding the publication of this paper.

\section{Acknowledgments}

The authors express their gratitude to the Ankara University BAUM Center for data acquisition and Cemre Topcu for assistance with stimulus delivery. This study was funded by Higher Education Council of Turkey (YÖK-Yükseköğretim Kurumu).

\section{References}

1. D. L. Dailey et al., "Transcutaneous electrical nerve stimulation reduces pain, fatigue and hyperalgesia while restoring central inhibition in primary fibromyalgia," Pain 154(11), 2554-2562 (2013).

2. D. J. Clauw, "Fibromyalgia: a clinical review," J. Am. Med. Assoc. 311(15), 1547-1555 (2014).

3. R. Staud et al., "Enhanced central pain processing of fibromyalgia patients is maintained by muscle afferent input: a randomized, double-blind, placebo-controlled study," Pain 145(1-2), 96-104 (2009).

4. M. Lofgren and C. Norrbrink, "Pain relief in women with fibromyalgia: a cross-over study of superficial warmth stimulation and transcutaneous electrical nerve stimulation," J. Rehabil. Med. 41(7), 557-562 (2009).

5. F. Carbonario et al., "Effectiveness of high-frequency transcutaneous electrical nerve stimulation at tender points as adjuvant therapy for patients with fibromyalgia," Eur. J. Phys. Rehabil. Med. 49(2), 197204 (2013).

6. G. R. Lauretti, E. F. Chubaci, and A. L. Mattos, "Efficacy of the use of two simultaneously TENS devices for fibromyalgia pain," Rheumatol. Int. 33(8), 2117-2122 (2013).

7. B. Mutlu et al., "Efficacy of supervised exercise combined with transcutaneous electrical nerve stimulation in women with fibromyalgia: a prospective controlled study," Rheumatol. Int. 33(3), 649-655 (2013).

8. R. Melzack and P. D. Wall, "Pain mechanisms: a new theory," Science 150(3699), 971-979 (1965).

9. Y. Maeda et al., "Release of GABA and activation of GABA(A) in the spinal cord mediates the effects of TENS in rats," Brain Res 1136(1), 43-50 (2007).

10. J. M. DeSantana et al., "Transcutaneous electrical nerve stimulation at both high and low frequencies activates ventrolateral periaqueductal grey to decrease mechanical hyperalgesia in arthritic rats," Neuroscience 163(4), 1233-1241 (2009).

11. K. A. Sluka, C. G. Vance, and T. L. Lisi, "High-frequency, but not lowfrequency, transcutaneous electrical nerve stimulation reduces aspartate 
and glutamate release in the spinal cord dorsal horn," J. Neurochem. 95(6), 1794-1801 (2005).

12. R. Peyron, B. Laurent, and L. Garcia-Larrea, "Functional imaging of brain responses to pain. A review and meta-analysis (2000)," Neurophysiol. Clin. 30(5), 263-288 (2000).

13. A. V. Apkarian et al., "Human brain mechanisms of pain perception and regulation in health and disease," Eur. J. Pain 9(4), 463 (2005).

14. Z. Barati, I. Zakeri, and K. Pourrezaei, "Functional near-infrared spectroscopy study on tonic pain activation by cold pressor test," Neurophotonics 4(1), 015004 (2017).

15. A. Pourshoghi, I. Zakeri, and K. Pourrezaei, "Application of functional data analysis in classification and clustering of functional near-infrared spectroscopy signal in response to noxious stimuli," J. Biomed. Opt. 21(10), 101411 (2016).

16. A. Vrana et al., "Cortical sensorimotor processing of painful pressure in patients with chronic lower back pain-an optical neuroimaging study using fNIRS," Front Hum. Neurosci. 10, 578 (2016).

17. C. M. Aasted et al., "Frontal lobe hemodynamic responses to painful stimulation: a potential brain marker of nociception," PLoS One 11(11), e0165226 (2016).

18. L. Becerra et al., "Diffuse optical tomography activation in the somatosensory cortex: specific activation by painful vs. non-painful thermal stimuli," PLoS One 4(11), e8016 (2009).

19. L. Becerra et al., "Diffuse optical tomography of pain and tactile stimulation: activation in cortical sensory and emotional systems," NeuroImage 41(2), 252-259 (2008)

20. N. Uceyler et al., "Increased cortical activation upon painful stimulation in fibromyalgia syndrome," BMC Neurol. 15, 210 (2015).

21. C. A. Porro et al., "Functional activity mapping of the mesial hemispheric wall during anticipation of pain," Neurolmage 19(4), 17381747 (2003)

22. J. C. Choi et al., "Brain mechanisms of pain relief by transcutaneous electrical nerve stimulation: a functional magnetic resonance imaging study," Eur. J. Pain 20(1), 92-105 (2016).

23. U. Bingel et al., "Subcortical structures involved in pain processing: evidence from single-trial fMRI," Pain 99(1-2), 313-321 (2002).

24. U. Bingel et al., "Single trial fMRI reveals significant contralateral bias in responses to laser pain within thalamus and somatosensory cortices," NeuroImage 18(3), 740-748 (2003).

25. L. L. Symonds et al., "Right-lateralized pain processing in the human cortex: an FMRI study," J. Neurophysiol. 95(6), 3823-3830 (2006).

26. J. C. Brooks et al., "fMRI of thermal pain: effects of stimulus laterality and attention," NeuroImage 15(2), 293-301 (2002)

27. R. H. Gracely et al., "Functional magnetic resonance imaging evidence of augmented pain processing in fibromyalgia," Arthritis Rheumatol. 46(5), 1333-1343 (2002).

28. D. B. Cook et al., "Functional imaging of pain in patients with primary fibromyalgia," J. Rheumatol. 31(2), 364-378 (2004).

29. T. Giesecke et al., "Evidence of augmented central pain processing in idiopathic chronic low back pain," Arthritis Rheumatol. 50(2), 613-623 (2004).

30. R. H. Gracely et al., "Pain catastrophizing and neural responses to pain among persons with fibromyalgia," Brain 127(Pt 4), 835-843 (2004).

31. T. Giesecke et al., "The relationship between depression, clinical pain, and experimental pain in a chronic pain cohort," Arthritis Rheumatol. 52(5), 1577-1584 (2005).

32. M. Burgmer et al., "Altered brain activity during pain processing in fibromyalgia," Neurolmage 44(2), 502-508 (2009).

33. J. Pujol et al., "Mapping brain response to pain in fibromyalgia patients using temporal analysis of FMRI," PLoS One 4(4), e5224 (2009).

34. M. Burgmer et al., "Fibromyalgia unique temporal brain activation during experimental pain: a controlled fMRI study," J. Neural Transm. 117(1), 123-131 (2010).

35. K. B. Jensen et al., "Anxiety and depressive symptoms in fibromyalgia are related to poor perception of health but not to pain sensitivity or cerebral processing of pain," Arthritis Rheumatol. 62(11), 3488-3495 (2010).

36. M. Lopez-Sola et al., "Altered functional magnetic resonance imaging responses to nonpainful sensory stimulation in fibromyalgia patients," Arthritis Rheumatol. 66(11), 3200-3209 (2014).

37. N. Fallon et al., "Altered cortical processing of observed pain in fibromyalgia syndrome patients," J. Pain 16(8), 717-726 (2015).
38. D. Pud, Y. Golan, and R. Pesta, "Hand dominancy—a feature affecting sensitivity to pain," Neurosci. Lett. 467(3), 237-240 (2009).

39. M. Ferrari and V. Quaresima, "A brief review on the history of human functional near-infrared spectroscopy (fNIRS) development and fields of application," NeuroImage 63(2), 921-935 (2012).

40. D. A. Boas et al., "Twenty years of functional near-infrared spectroscopy: introduction for the special issue," Neurolmage 85(Part 1), $1-5$ (2014).

41. C. J. Woolf, "Central sensitization: implications for the diagnosis and treatment of pain," Pain 152(3 Suppl.), S2-S15 (2011).

42. F. Wolfe et al., "The American College of Rheumatology 1990 criteria for the classification of fibromyalgia. Report of the Multicenter Criteria Committee," Arthritis Rheumatol. 33(2), 160-172 (1990).

43. R. Ambalavanar et al., "Deep tissue inflammation upregulates neuropeptides and evokes nociceptive behaviors which are modulated by a neuropeptide antagonist," Pain 120(1-2), 53-68 (2006).

44. B. Tena et al., "Reproducibility of electronic von Frey and von Frey monofilaments testing," Clin. J. Pain 28(4), 318-323 (2012).

45. C. Sparks et al., "Using functional magnetic resonance imaging to determine if cerebral hemodynamic responses to pain change following thoracic spine thrust manipulation in healthy individuals," J. Orthop. Sports Phys. Ther. 43(5), 340-348 (2013).

46. M. Cope and D. T. Delpy, "System for long-term measurement of cerebral blood and tissue oxygenation on newborn infants by near infra-red transillumination," Med. Biol. Eng. Comput. 26(3), 289-294 (1988).

47. H. H. Jasper, "The ten twenty electrode system of the international federation," Electroencephalogr. Clin. Neurophysiol. 10, 371-375 (1958).

48. L. Koessler et al., "Automated cortical projection of EEG sensors: anatomical correlation via the international 10-10 system," Neurolmage 46(1), 64-72 (2009)

49. M. Okamoto et al., "Three-dimensional probabilistic anatomical cranio-cerebral correlation via the international 10-20 system oriented for transcranial functional brain mapping," NeuroImage 21(1), 99-111 (2004).

50. T. Fekete et al., "The NIRS analysis package: noise reduction and statistical inference," PLoS One 6(9), e24322 (2011).

51. D. Tsuzuki and I. Dan, "Spatial registration for functional near-infrared spectroscopy: from channel position on the scalp to cortical location in individual and group analyses," NeuroImage 85(Pt 1), 92-103 (2014).

52. D. W. Shattuck et al., "Construction of a 3D probabilistic atlas of human cortical structures," Neurolmage 39(3), 1064-1080 (2008).

53. K. E. Jang et al., "Wavelet minimum description length detrending for near-infrared spectroscopy," J. Biomed. Opt. 14(3), 034004 (2009).

54. M. L. Schroeter et al., "Near-infrared spectroscopy can detect brain activity during a color-word matching Stroop task in an event-related design," Hum. Brain Mapp. 17(1), 61-71 (2002).

55. I. Jones and M. I. Johnson, "Transcutaneous electrical nerve stimulation," Contin. Educ. Anaesth., Crit. Care Pain 9(4), 130-135 (2009).

56. S. Lolignier, N. Eijkelkamp, and J. N. Wood, "Mechanical allodynia," Pflugers Arch. 467(1), 133-139 (2015).

57. K. B. Jensen et al., "Patients with fibromyalgia display less functional connectivity in the brain's pain inhibitory network," Mol. Pain 8, 32 (2012).

58. E. M. Pogatzki-Zahn, J. S. Englbrecht, and S. A. Schug, "Acute pain management in patients with fibromyalgia and other diffuse chronic pain syndromes," Curr. Opin. Anaesthesiol. 22(5), 627-633 (2009).

59. A. Ozcan et al., "Comparison of pressure pain threshold, grip strength, dexterity and touch pressure of dominant and non-dominant hands within and between right-and left-handed subjects," J. Korean Med. Sci. 19(6), 874-878 (2004).

60. P. Pauli, G. Wiedemann, and M. Nickola, "Pressure pain thresholds asymmetry in left- and right-handers: associations with behavioural measures of cerebral laterality," Eur. J. Pain 3(2), 151-156 (1999).

61. R. Chandramouli, B. R. Kanchan, and B. Ambadevi, "Right-left asymmetry in tonic pain perception and its modification by simultaneous contralateral noxious stimulation," Neuropsychologia 31(7), 687-694 (1993).

62. W. G. Friedli, P. Fuhr, and W. Wiget, "Detection threshold for percutaneous electrical stimuli: asymmetry with respect to handedness," $J$. Neurol., Neurosurg. Psychiatry 50(7), 870-876 (1987).

63. J. D. Greenspan and S. L. McGillis, "Thresholds for the perception of pressure, sharpness, and mechanically evoked cutaneous pain: effects of 
laterality and repeated testing," Somatosens. Motor Res. 11(4), 311-317 (1994).

64. D. J. Taylor, S. L. McGillis, and J. D. Greenspan, "Body site variation of heat pain sensitivity," Somatosens. Motor Res. 10(4), 455-465 (1993).

65. M. Neri and E. Agazzani, "Aging and right-left asymmetry in experimental pain measurement," Pain 19(1), 43-48 (1984).

66. A. V. Newton and J. M. Mumford, "Lateral dominance, pain perception, and pain tolerance," J. Dent. Res. 51(4), 940-942 (1972).

67. F. S. Murray and J. F. Safferstone, "Pain threshold and tolerance of right and left hands," J. Comp. Physiol. Psychol. 71(1), 83-86 (1970).

68. H. M. Buchanan and J. A. Midgley, "Evaluation of pain threshold using a simple pressure algometer," Clin. Rheumatol. 6(4), 510-517 (1987).

69. J. Brennum et al., "Measurements of human pressure-pain thresholds on fingers and toes," Pain 38(2), 211-217 (1989).

70. R. Jensen et al., "Cephalic muscle tenderness and pressure pain threshold in a general population," Pain 48(2), 197-203 (1992).

71. K. L. Petersen, J. Brennum, and J. Olesen, "Evaluation of pericranial myofascial nociception by pressure algometry. Reproducibility and factors of variation," Cephalalgia 12(1), 33-37 (1992).

72. B. B. Schiff and L. Gagliese, "The consequences of experimentally induced and chronic unilateral pain: reflections of hemispheric lateralization of emotion," Cortex 30(2), 255-267 (1994).

73. E. Sarlani, N. Farooq, and J. D. Greenspan, "Gender and laterality differences in thermosensation throughout the perceptible range," Pain 106(1-2), 9-18 (2003).

74. E. G. Duerden and M. C. Albanese, "Localization of pain-related brain activation: a meta-analysis of neuroimaging data," Hum. Brain Mapp. 34(1), 109-149 (2013).

75. F. Moran et al., "Hypoalgesia in response to transcutaneous electrical nerve stimulation (TENS) depends on stimulation intensity," J. Pain 12(8), 929-935 (2011).

Aykut Eken received his BS and MS degrees in biomedical engineering from Baskent University and his PhD in medical informatics from the Middle East Technical University, Ankara, Turkey. He is a research assistant doctor in the Biomedical Engineering Department at Düzce University. His research interests are neuroimaging, neural signal processing, and machine learning applications in neuroscience.

Murat Kara is an associate professor in the Department of Physical and Rehabilitation Medicine at Hacettepe University Medical School, Ankara, Turkey. He has finished medical training and residency in the Department of Physical and Rehabilitation Medicine at Hacettepe University Medical School, Ankara, Turkey. His main areas of interest comprise musculoskeletal ultrasonography, scientific publishing, electromyography, and pain.

Bora Baskak graduated from Ankara University Medical School and finished his residency on psychiatry at Ankara University. He is a fulltime associate professor in the Psychiatry Department at Ankara University Medical School. He is also the director of the fNIRS Laboratory, Ankara University Brain Research Center. His is interested in psychosis research, particularly neuroimaging in psychosis and psychopharmacology.

Ayşegül Baltacı graduated from Ege University, Medical Faculty, Izmir, Turkey, in 2006. She is a MD in the Department of Physical Medicine and Rehabilitation at Yenimahalle Teaching and Research Hospital. She completed physical medicine and rehabilitation residency program at Physical Medicine and Rehabilitation Education and Research Hospital, Ankara, in 2013. Her special interests include spinal cord injury, rheumatology, and algology.

Didem Gökçay received her BS and MS degrees in electrical engineering from the Middle East Technical University and her PhD in computer and information science and engineering from the University of Florida. She was a fulbright scholar during her PhD studies. She worked as a research fellow at the University of California San Diego. Her expertise areas are neuroimaging and affective neuroscience. Currently, she is an assistant professor in the Department of Medical Informatics at Middle East Technical University. 